\title{
Research Article Traffic Behavior in CA Model of Vehicular Traffic through a Series of Signals
}

\author{
Kazuhiro Tobita and Takashi Nagatani \\ Department of Mechanical Engineering, Shizuoka University, Hamamatsu 432-8561, Japan \\ Correspondence should be addressed to Takashi Nagatani, tmtnaga@ipc.shizuoka.ac.jp
}

Received 11 September 2012; Accepted 14 November 2012

Academic Editor: Wuhong Wang

Copyright (C) 2012 K. Tobita and T. Nagatani. This is an open access article distributed under the Creative Commons Attribution License, which permits unrestricted use, distribution, and reproduction in any medium, provided the original work is properly cited.

We study the traffic states and fundamental diagram of vehicular traffic controlled by a series of traffic lights using a deterministic cellular automaton (CA) model. The CA model is not described by a set of rules but is given by a difference equation. The vehicular traffic varies highly with both signal's characteristics and vehicular density. The dependence of fundamental diagram on the signal's characteristics is derived. At a low value of cycle time, the fundamental diagram displays the typical trapezoid, while it shows a triangle at a high value of cycle time. The dynamic transitions among distinct traffic states depend greatly on the signal's characteristics. The dependence of the transition points on the cycle time split and offset time is found.

\section{Introduction}

Mobility is nowadays one of the most significant ingredients of a modern society. Recently, transportation problems have attracted much attention in the fields of physics [1-4]. Physics and other sciences meet at the frontier area of interdisciplinary research. The traffic flow, pedestrian flow, and bus-route problem have been studied from a point of view of statistical mechanics and nonlinear dynamics [5-7]. The interesting dynamic behaviors have been found in the transportation system. The jams, chaos, and pattern formation are typical signatures of the complex behavior of transportation $[8,9]$. The cellular automaton (CA) model has been used extensively for the traffic dynamics [1,3]. The CA model for traffic flow is a typical system of discrete dynamics.

The traffic light is an essential element for managing the transportation network. In urban traffic, vehicles are controlled by traffic lights to give priority for a road because the city traffic networks often exceed the capacity and one avoids a collision between vehicles. Brockfeld et al. have studied optimizing traffic lights for city traffic by using a CA traffic 
model [10]. They have clarified the effect of signal control strategy on vehicular traffic. Also, they have shown that the city traffic controlled by traffic lights can be reduced to a simpler problem of a single-lane roadway. D. W. Huang and W. N. Huang have studied the traffic flow controlled by signals on a single-lane roadway by using Nagel-Schreckenberg model [11]. Sasaki and Nagatani have investigated the traffic flow on a single-lane roadway with traffic lights by using the optimal velocity model [12]. They have derived the relationship between the road capacity and jamming transition. Until now, one has studied the periodic traffic controlled by a few traffic lights. It has been concluded that the periodic traffic does not depend on the number of traffic lights. Very recently, Lammer and Helbing have studied the vehicular flow by the self-control of traffic signals in urban road networks [13].

In real traffic, the vehicular traffic depends highly on the configuration of traffic lights and the priority of roadways. In the dilute limit of vehicular density, a few works have been done for the traffic of vehicles moving through an infinite series of traffic lights with the same interval. The effect of cycle time on vehicular traffic has been clarified by using the nonlinear-map models [14-18]. Also, it has been shown that the heterogeneity of signal's interval and irregular split have the important effects on vehicular traffic [19].

The deterministic CA model has been presented for the vehicular traffic through a series of traffic lights at the synchronized strategy for various values of vehicular density [20]. The dependence of the travel time on both cycle time and density has been clarified. The effect of a slow vehicle on the traffic flow through a series of signals has been investigated [21]. Also, it has been shown that the fundamental diagram depends a little on the cycle time for an intermediate value of the cycle time [20]. It has been found that the dynamic transition occurs from the undersaturated traffic, through the saturated traffic, to the oversaturated traffic [20]. However, it is little known how the dynamic transition depends on the signal' characteristics for the traffic flow through a series of signals. Also, it is unknown how the dynamic transition varies with the cycle time at the green-wave strategy. It will be necessary and important to study the dynamic transition and the fundamental diagram for the traffic flow through a series of signals at the green-wave strategy.

Until now, the traffic flow controlled by a few signals has been studied. Also, the traffic flow through the series of signals controlled by split 0.5 and zero offset has been investigated with the use of CA model. The effects of split and offset on traffic flow through the series of signals are known little. Also, the dependence of the fundamental diagram on the cycle time, the split, and the offset is unclear.

In order to study the effect of offset on the traffic flow, it is necessary to take into account the series of signals because the phase shift varies from signal to signal at the greenwave strategy.

In this paper, we take into account the split and offset. We clarify the effect of split and offset on the traffic flow. We study the fundamental diagram and dynamic transitions for traffic flow through a series of traffic lights at the synchronized and green-wave strategies by using the CA model described by the difference equation. We clarify the dependence of dynamic transition on the signal's characteristics. We show how the fundamental diagram and transition points vary with the cycle time, split, and phase shift of signals. Especially, we investigate the dynamic transitions and fundamental diagram at a high value of the cycle time. 


\section{CA Model and Difference Equation}

We consider the flow of vehicles going through the series of traffic lights on one-dimensional lattice. Each vehicle does not pass over other vehicles. The traffic lights are positioned homogeneously on a roadway. The interval between signals has a constant value and is given by $l$. All traffic lights change from red (green) to green (red) with a fixed time period $\left(1-s_{p}\right) t_{s}\left(s_{p} t_{s}\right)$. The period of green is $s_{p} t_{s}$ and the period of red is $\left(1-s_{p}\right) t_{s}$. Time $t_{s}$ is called the cycle time and fraction $s_{p}$ represents the split which indicates the ratio of green time to cycle time.

We apply the Fukui-Ishibashi model to the vehicular motion [22]. The Fukui-Ishibashi model is not stochastic but deterministic. We use the deterministic CA model in place of the stochastic Nagel-Schreckenberg model [3] because the dynamic transition occurs clearly in the deterministic model. We extend the deterministic CA model to take into account traffic lights. We define the position of vehicle $i$ at time $t$ as $x_{i}(t)$ where $x, i$, and $t$ are an integer. The velocity takes the integer value ranging from 0 to $v_{\max }$. The velocity depends on the headway. If headway $\Delta x_{i}(t)\left(=x_{i+1}(t)-x_{i}(t)\right)$ is larger than the maximum velocity, the vehicle moves with the maximum velocity. If the headway is less than the maximum velocity, the vehicle moves with velocity $\Delta x_{i}(t)-1$.

When a vehicle arrives at a traffic light and the traffic light is red, the vehicle stops at the position of the traffic light. Then, when the traffic light changes from red to green, the vehicle goes ahead. On the other hand, when a vehicle arrives at a traffic light and the traffic light is green, the vehicle does not stop and goes ahead without changing speed. The position of the closest signal before vehicle $i$ at time $t$ is given by

$$
x_{i, S}(t)=\left\{\operatorname{int}\left(\frac{x_{i}(t)}{l}\right)+1\right\} l
$$

We set split as $s_{p}=0.5$. Then, the position of vehicle $i$ at time $t+1$ is given by

$$
\begin{aligned}
x_{i}(t+1)= & \min \left[x_{i}(t)+v_{\max }, x_{i+1}(t)-1\right]\left\{1-\vartheta\left(\sin \left(\frac{2 \pi t}{t_{s}}\right)\right)\right\} \\
& +\min \left[x_{i}(t)+v_{\max }, x_{i+1}(t)-1, x_{i, s}(t)-1\right] \vartheta\left(\sin \left(\frac{2 \pi t}{t_{s}}\right)\right) .
\end{aligned}
$$

Here, $\vartheta(t)$ is the step function. It takes one if $t>0$ and zero if $t \leq 0 . \min [A, B]$ is a minimum function and takes the minimum value within $A$ and $B$.

If the signal just before vehicle $i$ is green, $\vartheta\left(\sin \left(2 \pi t / t_{s}\right)\right)=0$ and $x_{i}(t+1)=\min \left[x_{i}(t)+\right.$ $\left.v_{\max }, x_{i+1}(t)-1\right]$. Otherwise, if the signal just before vehicle $i$ is red, $\vartheta\left(\sin \left(2 \pi t / t_{s}\right)\right)=1$ and $x_{i}(t+1)=\min \left[x_{i}(t)+v_{\max }, x_{i+1}(t)-1, x_{i, s}(t)-1\right]$. Then, if the headway is larger than $v_{\max }$, vehicle $i$ stops at site $x_{i, s}(t)-1$ just before the signal. Also, if $x_{i+1}(t)$ is higher than $x_{i, s}(t)$, vehicle $i$ stops at site $x_{i, s}(t)-1$ just before the signal. Thus, (2.2) presents the CA model for the vehicular traffic through a series of traffic lights. Equation (2.2) is a single difference equation. Until now, the CA model for the signal traffic has been described by a set of CA rules. However, model (2.2) is of great advantage to simulate the traffic flow because the difference equation is simple.

We extend (2.2) to take into account the phase shift and split. The signal timing is controlled by offset time $t_{\text {offset }}$. The offset time means the difference of phase shifts between 


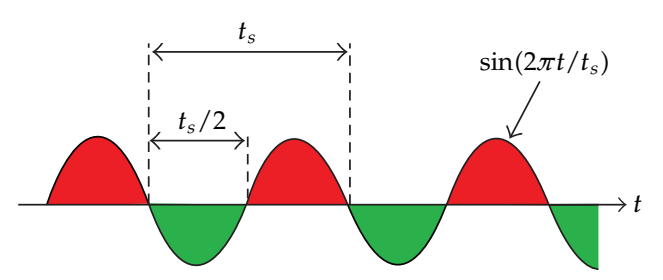

(a)

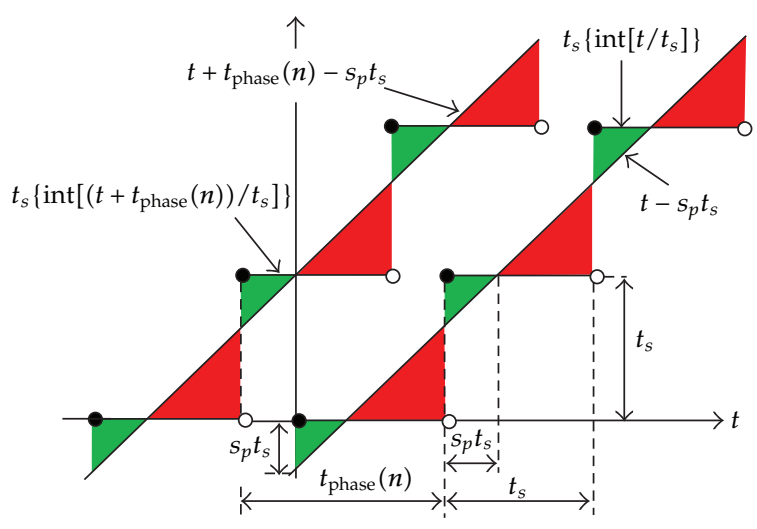

(b)

Figure 1: Schematic illustration of periodic functions. (a) Sine wave. (b) Generalized periodic function.

two successive signals. In the green wave (delayed) strategy, the phase shift of signal $n$ is given by $t_{\text {phase }}(n)=n t_{\text {offset }}$ where the phase shift is indicated by $t_{\text {phase }}(n)$. Then, the signal switches from red to green in green wave way. The phase shift increases with signal $n$.

The position of vehicle $i$ at time $t+1$ is given by

$$
\begin{aligned}
x_{i}(t+1)= & \min \left[x_{i}(t)+v_{\text {max }}, x_{i+1}(t)-1\right] \\
& \times\left\{1-\vartheta\left[t+t_{\text {phase }}(n)-t_{s}\left\{\operatorname{int}\left(\frac{t+t_{\text {phase }}(n)}{t_{s}}\right)\right\}-s_{p} t_{s}\right]\right\} \\
& +\min \left[x_{i}(t)+v_{\text {max }}, x_{i+1}(t)-1, x_{i, s}(t)-1\right] \\
& \times \vartheta\left[t+t_{\text {phase }}(n)-t_{s}\left\{\operatorname{int}\left(\frac{t+t_{\text {phase }}(n)}{t_{s}}\right)\right\}-s_{p} t_{s}\right] .
\end{aligned}
$$

Periodic function $\sin \left(2 \pi t / t_{s}\right)$ in $(2.2)$ is replaced by generalized periodic function $[t+$ $\left.t_{\text {phase }}(n)-t_{s}\left\{\operatorname{int}\left(\left(t+t_{\text {phase }}(n)\right) / t_{s}\right)\right\}-s_{p} t_{s}\right]$ in (2.3). In the generalized periodic function, the phase shifts are included explicitly. However, if one extends periodic function $\sin \left(2 \pi t / t_{s}\right)$ to take into account the split, one cannot include the split explicitly. Figure 1(a) shows the schematic illustration of traffic sign given by a sine wave in (2.2). The cycle time is given by $t_{s}$. The split is $s_{p}=0.5$. Figure $1(\mathrm{~b})$ shows the schematic illustration of the generalized periodic function in (2.3). The split is set explicitly by any value $s_{p}$. Also, the phase shift is displayed by $t_{\text {phase }}$. The green and red signs are illustrated by the green and red colors, respectively.

When $s_{p}=0.5$ and $t_{\text {phase }}=0,(2.3)$ reduces to (2.2). If there are no signals on the roadway, (2.3) reduces to the CA model of Fukui and Ishibashi

$$
x_{i}(t+1)=\min \left[x_{i}(t)+v_{\max }, x_{i+1}(t)-1\right]
$$

where $v_{\max }$ is the maximum velocity and an integer. 
It will be expected that the vehicular traffic exhibits a complex behavior by dynamic transitions. We study how the vehicular traffic changes by varying the cycle time, split, phase shift, and vehicular density.

Until now, CA models with no signals and with a few signals have been proposed. In the previous work [20], we have presented the CA model with the series of signals at the synchronized strategy. The split and the offset (phase shift) have been not taken into account. Generally, it is necessary and important to take into account the split and the offset (phase shift). In this study, we have proposed the CA model with the series of signals to take into account the split and the offset of signals. The conventional CA model is described in terms of the set of rules. However, our CA model is given by the difference equation (2.3). The difference equation (2.3) is the first for the traffic flow controlled by the series of signals.

\section{Simulation Result}

We investigate the fundamental diagram, the traffic states, and the dynamic transitions by using (2.3). First, we study the traffic flow at the synchronized strategy for the signal control. Second, we investigate the traffic flow controlled by signals at the green-wave strategy.

We consider the city traffic network controlled by traffic signals. There always exist signals at a crossing. Vehicles move ahead through a series of signals at a street. We simulate the traffic flow on a single-lane roadway with many signals. We set the direction of vehicle flow as $x$-axis. A signal changes alternately from green (red) to red (green). We ignore the yellow signal. We consider the periodic boundary for the vehicular flow on a single-lane roadway. When vehicles reach the right edge of the roadway, they return the left edge of the roadway. We calculate the positions of all vehicles using (2.3).

\subsection{Synchronized Strategy}

In the synchronized strategy, all signals change simultaneously. We calculate the current (flow) versus density for various values of cycle time $t_{s}$. We set the interval between signals as $l=40$ and the maximal velocity as $v_{\max }=4$. We define the dimensionless cycle time as $T_{s}=t_{s} v_{\max } / l$. It is the ratio of cycle time to the moving time between a signal and its next signal. This means that the unit of time is the moving time over the interval between two signals. It has an advantage that the dimensionless cycle time does not vary even if the interval between two signals or maximum velocity changes. Also, the dimensionless offset time is defined as $\tau=t_{\text {offset }} v_{\max } / l$. Figure 2(a) shows the plots of mean current against density for dimensionless cycle time $T_{s}=3.0$ where the split is $s_{p}=0.5$, the offset time is $\tau=0.0$, and the road length is $L=4000$. The solid curve indicates the current profile. For comparison, the current for the vehicular traffic with no signals is shown by the chain line. The current profile changes from the triangle to the trapezoid by adding the signals. The traffic states change from the undersaturated traffic, through the saturated traffic, to the oversaturated traffic. In the unsaturated traffic region between points $0-b$, the current increases with density, two distinct states occur, and the traffic state changes at point $a$. In the saturated traffic region between points $b-c$, the current keeps a constant value. In the oversaturated traffic region between points $c-1$, the current decreases with increasing density, two distinct states appear, and the traffic state changes at point $d$. Thus, there exist five distinct traffic states and dynamic transitions occur at four points $a-d$ for dimensionless cycle time $T_{S}=3.0$. 


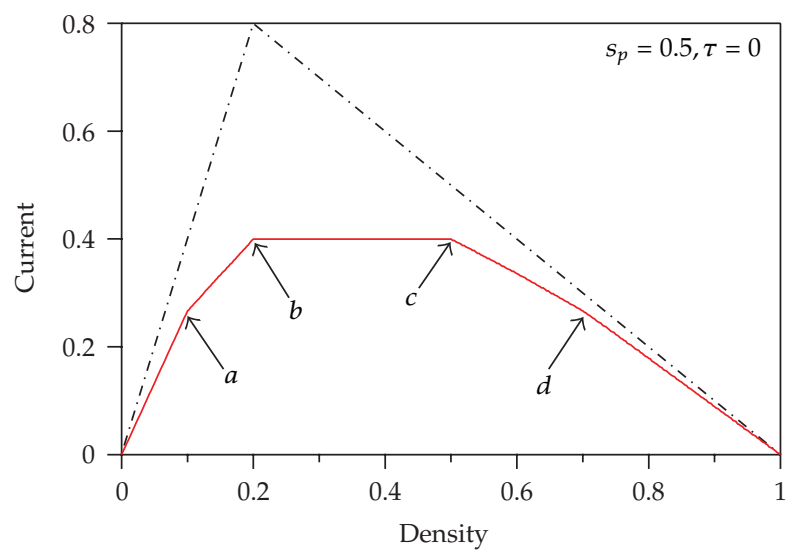

$-T_{\mathrm{s}}=3$

-..- No signal

(a)

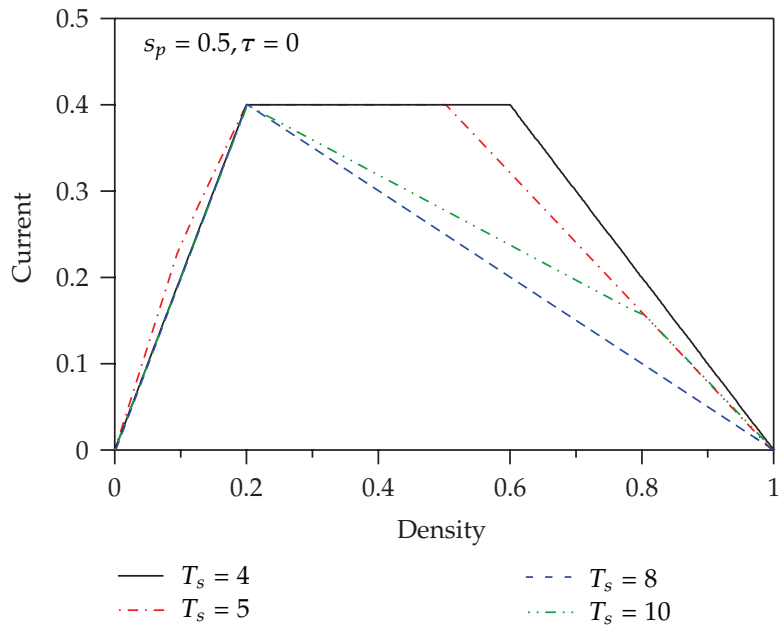

(b)

Figure 2: (a) Plots of mean current against density for $T_{s}=3.0$ where the split is $s_{p}=0.5$, the offset time is $\tau=0.0$, and the road length is $L=4000$. The solid curve indicates the current profile. For comparison, the current for the vehicular traffic with no signals is shown by the chain curve. (b) Fundamental diagrams for the dimensionless cycle times $T_{s}=4.0,5.0,8.0,10.0$.

We study the variation of fundamental diagram with the dimensionless cycle time. Figure 2(b) shows the fundamental diagrams for the dimensionless cycle times $T_{S}=4.0$, 5.0, 8.0, 10.0. The fundamental diagram depends greatly on the dimensionless cycle time. Especially, the fundamental diagram at high values of dimensionless cycle time is definitely different from that at low value of the cycle time. With increasing the cycle time, the current profile changes from the typical trapezoid to the triangle.

We study the traffic pattern (vehicular trajectories) for various values of density at dimensionless cycle time $T_{s}=3.0$. Figure 3 shows the trajectories of vehicles between $x=$ 400 and $x=600$ at various values of density for cycle time $T_{s}=3.0$ where the road length is $L=4000$, the interval between signals is $l=40$, and the maximal velocity is $v_{\max }=4$. 


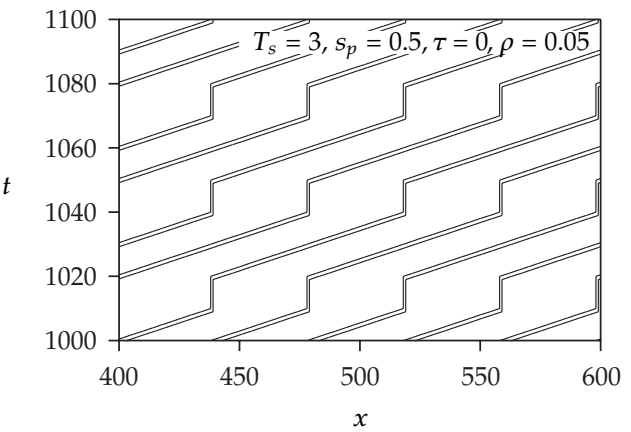

(a)

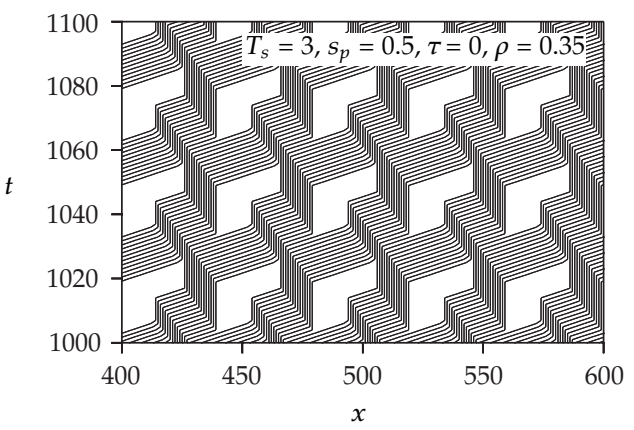

(c)

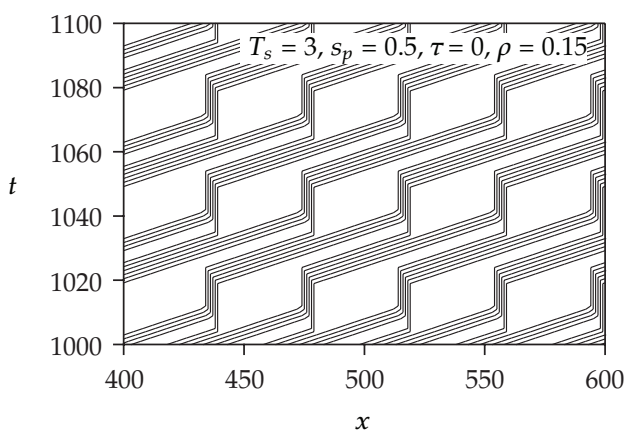

(b)

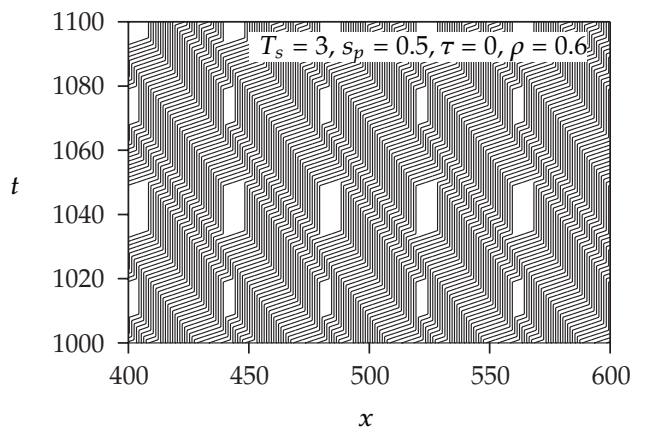

(d)

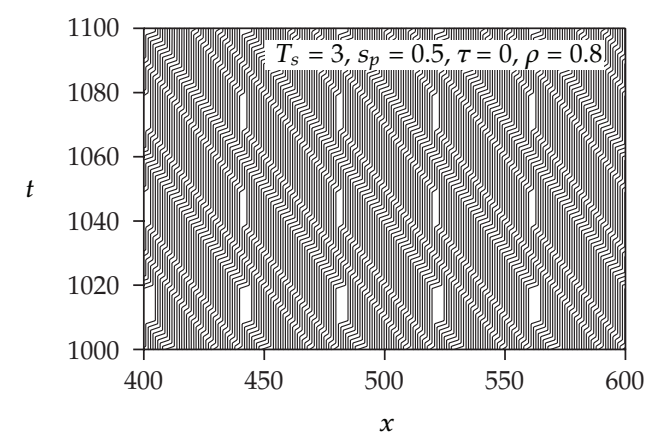

(e)

Figure 3: Trajectories of vehicles between $x=400$ and $x=600$ at various values of density for cycle time $T_{S}=3.0$ where the road length is $L=4000$, the interval between signals is $l=40$, and the maximal velocity is $v_{\max }=4$. Diagrams (a)-(e) are obtained, respectively, at densities (a) $\rho=0.05$, (b) $\rho=0.15$, (c) $\rho=0.35$, (d) $\rho=0.6$, and (e) $\rho=0.8$ for the fundamental diagram in Figure 2(a).

Diagrams (a)-(e) are obtained, respectively, at densities (a) $\rho=0.05$, (b) $\rho=0.15$, (c) $\rho=0.35$, (d) $\rho=0.6$, and (e) $\rho=0.8$ for the fundamental diagram in Figure 2(a). In diagram (a), a pair of two vehicles move together and stop every two signals. The traffic pattern (a) is typical for the traffic state at the region 0- $a$ in Figure 2(a). In diagram (b), a group of six vehicles move together. However, the group breaks up into two subgroups at a signal and merges again at the next to next signal. The breakup and coalescence are repeated. The traffic pattern (b) is typical for the traffic state at the region $a-b$ in Figure 2(a). The traffic state (a) changes to 
the traffic state (b) at transition point $b$ in Figure 2(a). In diagram (c), the stop- and go-wave is induced by stopping at the signal. The vehicles stop temporarily by the stop- and go-wave before they reach the signal. The stop- and go-wave disappears within the interval between two signals. In the traffic state (c), the traffic current saturates and keeps the maximum value. The traffic pattern (c) is typical for the traffic state at the region $b-c$ in Figure 2(a). At the transition point $b$, the traffic state (b) changes to the traffic state (c). In diagrams (d) and (e), the stop- and go-wave propagates backward through signals. In diagram (d), the region of the moving vehicles breaks up and coalescences, while the region of moving vehicles propagates backward with no breakup in diagram (e). At transition point $c$ in Figure 2(a), the traffic state (c) changes to the traffic state $(\mathrm{d})$. The traffic state (d) changes to the traffic state (e) at the transition point $d$. Thus, the traffic state changes at the transition points $a-d$. The vehicular trajectories change highly with density.

We study the dependence of the maximum current on the dimensionless cycle time. Also, we study the effect of the split $s_{p}$ on the maximum current $Q_{\max }$ and the dynamic transitions. Figure 4(a) shows the plot of the maximum currents $Q_{\max }$ against dimensionless cycle time $T_{s}$ at splits $s_{p}=0.25,0.5,0.75$. The squares, circles, and triangles indicate, respectively, the maximum currents at $s_{p}=0.25,0.5,0.75$. At split $s_{p}=0.5$, the maximum current goes up and down with increasing dimensionless cycle time and the width decreases with increasing dimensionless cycle time. The profile displays a saw-toothed shape. When $T_{s}$ approaches to an infinity, the maximum current approaches to 0.4. With increasing the split, the maximum current increases. When the split goes to infinity, the maximum currents at $s_{p}=0.25,0.5,0.75$ approach, respectively, to $0.2,0.4,0.6$. We rescale the profiles of the maximum current versus the dimensionless cycle time as Figure 4(b).

Figure $4(\mathrm{~b})$ shows the plots of rescaled maximum current $Q_{\max } / 2 s_{p}$ against rescaled dimensionless cycle time $T_{s} s_{p}$ for Figure 4(a). All rescaled maximum currents collapse on a single curve. The maximum current scales as

$$
Q_{\max }=2 s_{p} f\left(T_{s} s_{p}\right)
$$

where $f(x)$ is the scaling function.

In the synchronized strategy, the maximum current is proportional to the split. Also, the dependence of the maximum current on both cycle time and split is determined only by the product $T_{s} s_{p}$. It is important how the saturated traffic disappears with density. We study the dependence of transition points $b$ and $c$ on the dimensionless cycle time. Also, we study the dependence of transition points $b$ and $c$ on the split $s_{p}$. Figure 5(a) shows the plots of the transition densities $\rho_{c, b}$ and $\rho_{c, c}$ against dimensionless cycle time at splits $s_{p}=0.25,0.5,0.75$. The open squares, open circles, and open triangles indicate, respectively, the transition density $\rho_{c, b}$ of the dynamic transition from the undersaturated traffic to the saturated traffic at splits $s_{p}=0.25,0.5,0.75$. The full squares, full circles, and full triangles represent, respectively, the transition density $\rho_{c, c}$ of the dynamic transition from the saturated traffic to the oversaturated traffic at splits $s_{p}=0.25,0.5,0.75$.

At split $s_{p}=0.5$, the transition point $\rho_{c, b}$ keeps constant value 0.2 for $T_{s}>1.8$. The transition point $\rho_{c, c}$ varies up and down for $T_{s}<4$, then decreases linearly with increasing $T_{s}$, and keeps the constant value 0.2 for $T_{s}>7.8$. The transition point $\rho_{c, c}$ coalescences with the transition point $\rho_{c, b}$ at $T_{s}=7.8$. The saturated traffic disappears at $T_{s}=7.8$ for $s_{p}=0.5$. In the result, the fundamental diagram changes from the trapezoid to the triangle at dimensionless cycle time $T_{s}=7.8$. We rescale the profiles of the transition points versus 


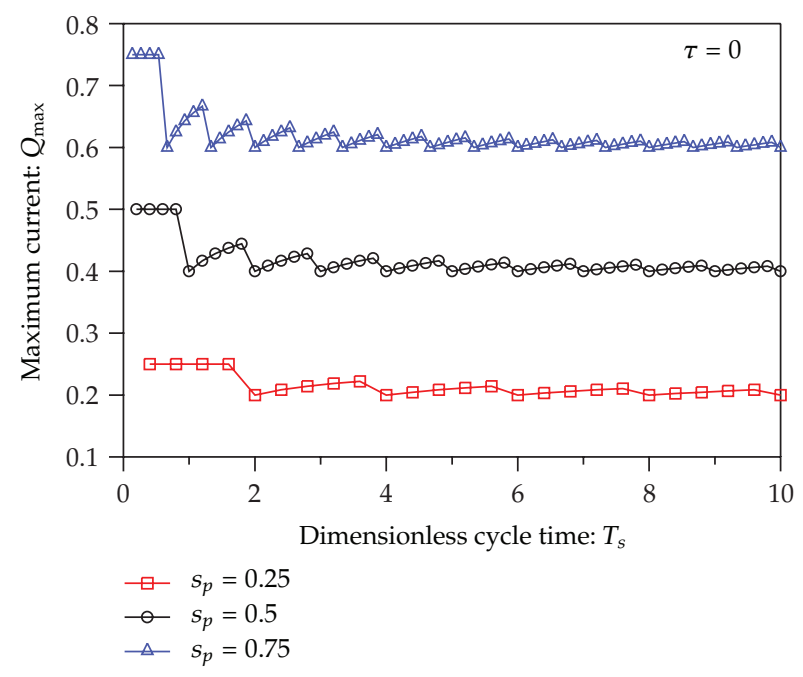

(a)

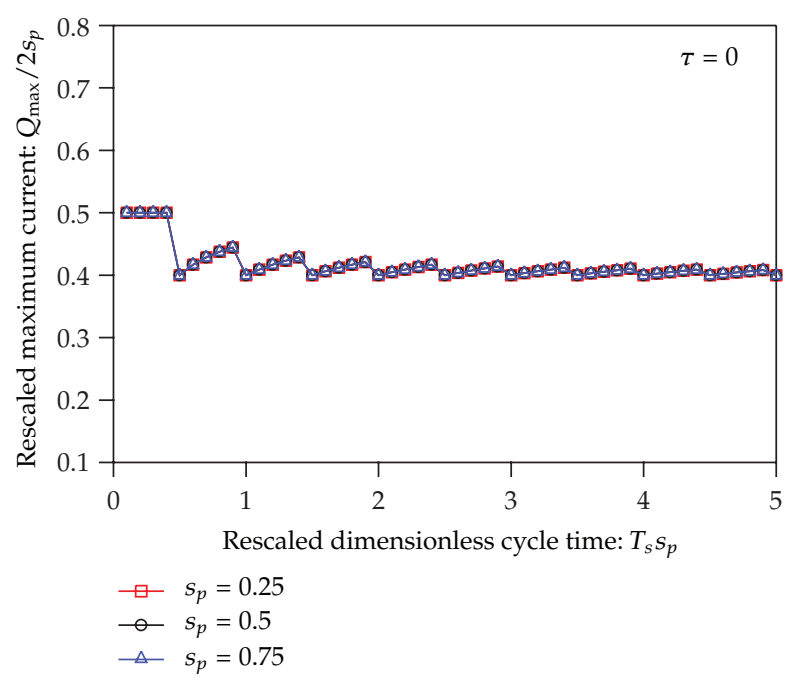

(b)

Figure 4: (a) Plot of the maximum currents $Q_{\max }$ against dimensionless cycle time $T_{s}$ at splits $s_{p}=0.25,0.5$, 0.75 . The squares, circles, and triangles indicate, respectively, the maximum currents at $s_{p}=0.25,0.5,0.75$. (b) Plots of rescaled maximum current $Q_{\max } / 2 s_{p}$ against rescaled dimensionless cycle time $T_{s} s_{p}$ for (a).

the dimensionless cycle time as Figure 5(b). Figure 5(b) shows the plots of transition points $\rho_{c, b}$ and $\rho_{c, c}$ against rescaled dimensionless cycle time $T_{s} s_{p}$ for Figure 5(a). The transition points $\rho_{c, b}$ at $s_{p}=0.25,0.5,0.75$ collapse on a single curve. Also, the transition points $\rho_{c, c}$ at $s_{p}=0.25,0.5,0.75$ collapse on a single curve. The transition points $\rho_{c, b}$ and $\rho_{c, c}$ scale as

$$
\begin{aligned}
& \rho_{c, b}=g_{1}\left(T_{s} s_{p}\right), \\
& \rho_{c, c}=g_{2}\left(T_{s} s_{p}\right),
\end{aligned}
$$

where $g_{1}(x)$ and $g_{2}(x)$ are the scaling functions for the two dynamic transitions. 


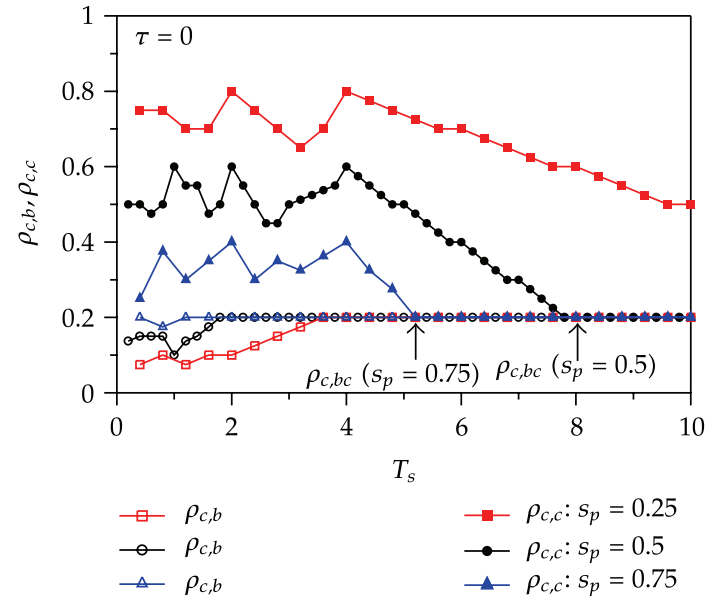

(a)

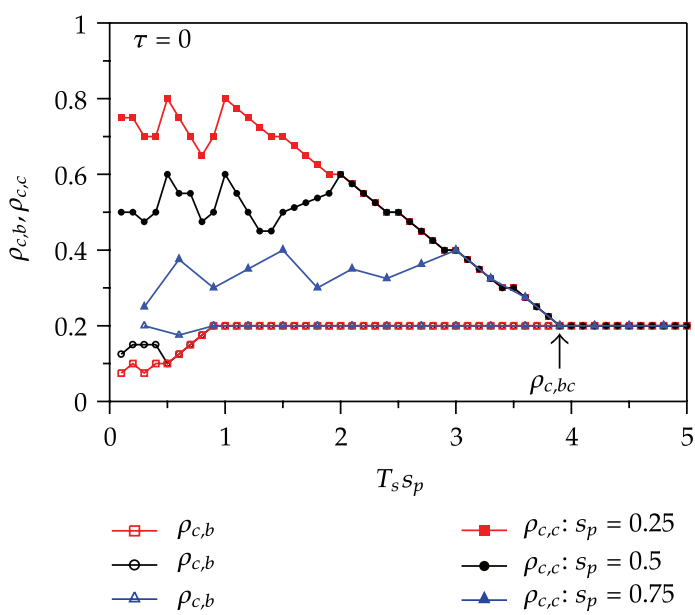

(b)

Figure 5: (a) Plots of the transition densities $\rho_{c, b}$ and $\rho_{c, c}$ against dimensionless cycle time $T_{s}$ at splits $s_{p}=0.25,0.5,0.75$. The open squares, open circles, and open triangles indicate, respectively, the transition density $\rho_{c, b}$ of the dynamic transition from the undersaturated traffic to the saturated traffic at splits $s_{p}=$ $0.25,0.5,0.75$. The full squares, full circles, and full triangles represent, respectively, the transition density $\rho_{c, c}$ of the dynamic transition from the saturated traffic to the oversaturated traffic at splits $s_{p}=0.25,0.5$, 0.75. (b) Plots of transition points $\rho_{c, b}$ and $\rho_{c, c}$ against rescaled dimensionless cycle time $T_{s} s_{p}$ for (a).

In the synchronized strategy, the transition points $\rho_{c, b}$ and $\rho_{c, c}$ depend only on the rescaled cycle time $T_{s} s_{p}$. If the product $T_{s} s_{p}$ of dimensionless cycle time $T_{s}$ by split $s_{p}$ is the same value, the dynamic transition between the undersaturated traffic and the saturated traffic occurs at the same value of the transition density. Also, when the product $T_{s} s_{p}$ of dimensionless cycle time $T_{s}$ by split $s_{p}$ is the same value, the dynamic transition between the saturated traffic and the oversaturated traffic occurs at the same value of the transition density. Thus, the dynamic transitions are governed only by the product $T_{s} s_{p}$.

\subsection{Green-Wave Strategy}

We study the traffic flow controlled by signals at the green-wave strategy. We investigate the effect of the offset time (phase shift difference) on the traffic flow. Signals vary from the upstream to the downstream and vice versa with constant value $t_{\text {offset }}$ of the phase shift difference. We define the dimensionless offset time as $\tau=t_{\text {offset }} v_{\max } / l$. We calculate the current (flow) versus density for various values of cycle time $t_{s}$ at dimensionless offset time $\tau=1.0$. We set the interval between signals as $l=40$ and the maximal velocity as $v_{\max }=4$. The dimensionless cycle time is defined as $T_{s}=t_{s} v_{\max } / l$. Figure 6(a) shows the plots of mean current against density at offset time $\tau=1.0$ and $T_{s}=3.0$ where the split is $s_{p}=0.5$ and the road length is $L=4000$. The solid curve indicates the current profile. For comparison, the current for the vehicular traffic with no signals is shown by the chain curve. Figure 6(a) is compared with Figure 2(a) at the synchronized strategy. The traffic states change from the undersaturated traffic, through the saturated traffic, to the oversaturated traffic. In the unsaturated traffic region between points $0-b$, the current increases with density, only a state 


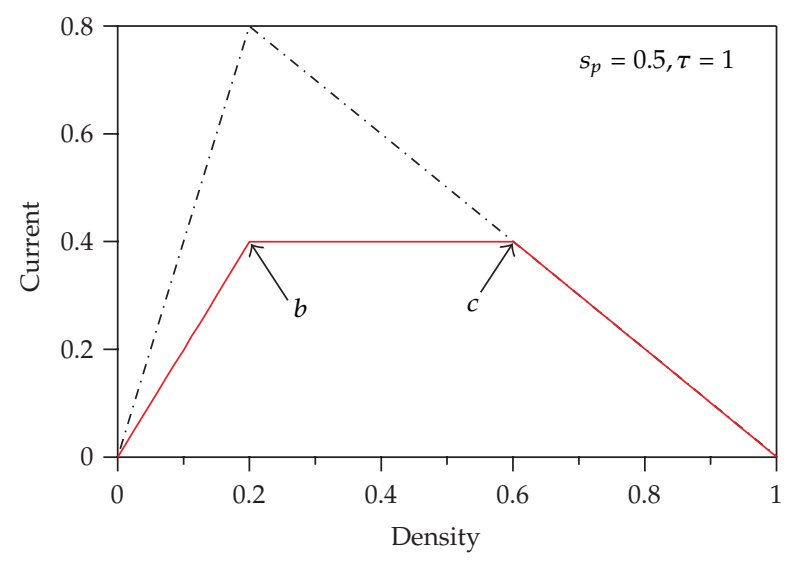

$-T_{s}=3$

...- No signal

(a)

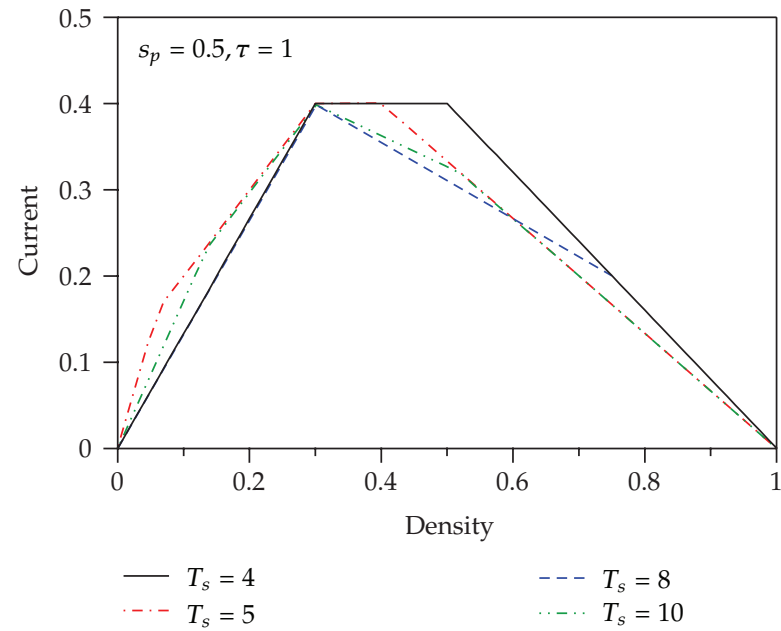

(b)

Figure 6: (a) Plots of mean current against density at offset time and where the split is $s_{p}=0.5$ and the road length is $L=4000$. The solid curve indicates the current profile. For comparison, the current for the vehicular traffic with no signals is shown by the chain curve. (b) Fundamental diagrams for the dimensionless cycle times.

occurs, and the traffic state does not change at point $a$ in Figure 2(a). In the saturated traffic region between points $b-c$, the current keeps a constant value. In the oversaturated traffic region between points $c-1$, the traffic state does not change at point $d$ in Figure 2(a). Thus, there exist three distinct traffic states and dynamic transitions occur at two points $b$ and $c$ for dimensionless cycle time $T_{S}=3.0$. Thus, the fundamental diagram in Figure 2(a) for the synchronized strategy changes to that in Figure 6(a) for $\tau=1.0$.

We study the variation of fundamental diagram at $\tau=1.0$ with the dimensionless cycle time. Figure 6(b) shows the fundamental diagrams at $\tau=1.0$ for the dimensionless cycle times $T_{s}=4.0,5.0,8.0,10.0$. Figure 6(b) is compared with Figure 2(b) at the synchronized strategy. The fundamental diagram depends greatly on the dimensionless cycle time. 
Especially, the fundamental diagram at high values of dimensionless cycle time is definitely different from that at low value of the cycle time. With increasing the cycle time, the current profile changes from the typical trapezoid to the triangle. However, the dependence of transition densities on the dimensionless cycle time is definitely different from that in Figure 2(b) at the synchronized strategy.

We study the dependence of transition points $b$ and $c$ on the split $s_{p}$ at $\tau=1.0$. Figure 7(a) shows the plots of the transition densities $\rho_{c, b}$ and $\rho_{c, c}$ against dimensionless cycle time at splits $s_{p}=0.25,0.5,0.75$ at $\tau=1.0$. The open squares, open circles, and open triangles indicate, respectively, the transition density $\rho_{c, b}$ of the dynamic transition from the undersaturated traffic to the saturated traffic at splits $s_{p}=0.25,0.5,0.75$. The full squares, full circles, and full triangles represent, respectively, the transition density $\rho_{c, c}$ of the dynamic transition from the saturated traffic to the oversaturated traffic at splits $s_{p}=0.25$, $0.5,0.75$. We rescale the profiles of the transition points versus the dimensionless cycle time as Figure $7(\mathrm{~b})$. Figure $7(\mathrm{~b})$ shows the plots of rescaled transition points $\rho_{c, b}-\left(1-s_{p}\right) / 5$ and $\rho_{c, c}-\left(1-s_{p}\right) / 5$ against rescaled dimensionless cycle time $T_{s} s_{p}$ for Figure $7(\mathrm{a})$. The rescaled transition points scale as those at the synchronized strategy in Figure 5(b). The transition points $\rho_{c, b}$ at $s_{p}=0.25,0.5,0.75$ collapse on a single curve. Also, the transition points $\rho_{c, c}$ at $s_{p}=0.25,0.5,0.75$ collapse on a single curve. The transition points $\rho_{c, b}$ and $\rho_{c, c}$ scale as

$$
\begin{aligned}
& \rho_{c, b}=\frac{1-s_{p}}{5}+g_{3}\left(T_{s} s_{p}\right), \\
& \rho_{c, c}=\frac{1-s_{p}}{5}+g_{4}\left(T_{s} s_{p}\right),
\end{aligned}
$$

where $g_{3}(x)$ and $g_{4}(x)$ are the scaling functions for the two dynamic transitions.

All rescaled transition points keep 0.2 for $T_{s} s_{p} \geq 3.0$. The fundamental diagram changes from the trapezoid to the triangle at point $\rho_{c, b c}\left(T_{s} s_{p}=3.0\right)$.

We calculate the current (flow) versus density for various values of cycle time $t_{s}$ at a negative value of dimensionless offset time $\tau=-1.0$. Figure 8 shows the fundamental diagrams at $\tau=-1.0$ for the dimensionless cycle times $T_{s}=4.0,5.0,8.0,10.0$. Figure 8 is compared with Figure 2(b) at the synchronized strategy and Figure 6(b) at $\tau=1.0$. The fundamental diagram depends greatly on the dimensionless offset time $\tau$. The fundamental diagram at $\tau=-1.0$ is definitely different from that at $\tau=0.0$ and that at $\tau=1.0$. With increasing the cycle time, the current profile changes from the typical trapezoid to the triangle. However, the dependence of transition densities on the dimensionless cycle time is definitely different from that in Figure 6(b) at $\tau=1.0$.

We study the dependence of transition points $b$ and $c$ on the split $s_{p}$ at $\tau=-1.0$. Figure 9(a) shows the plots of the transition densities $\rho_{c, b}$ and $\rho_{c, c}$ against dimensionless cycle time at splits $s_{p}=0.25,0.5,0.75$ at $\tau=-1.0$. We rescale the profiles of the transition points versus the dimensionless cycle time as Figure 9(b). Figure 9(b) shows the plots of rescaled transition points $\rho_{c, b}+\left(1-s_{p}\right) / 5$ and $\rho_{c, c}+\left(1-s_{p}\right) / 5$ against rescaled dimensionless cycle time $T_{s} s_{p}$ for Figure 9 (a). The transition points $\rho_{c, b}$ at $s_{p}=0.25,0.5,0.75$ collapse on a single 


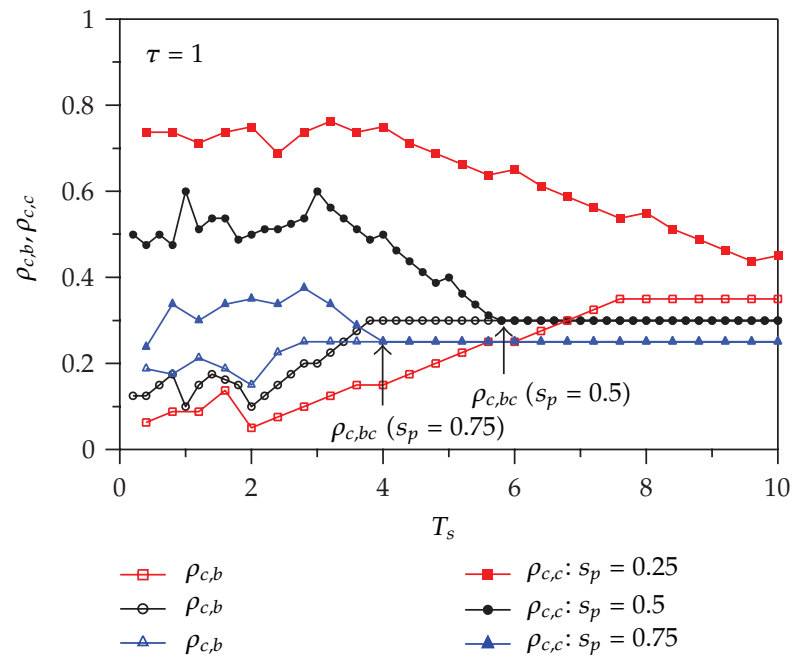

(a)

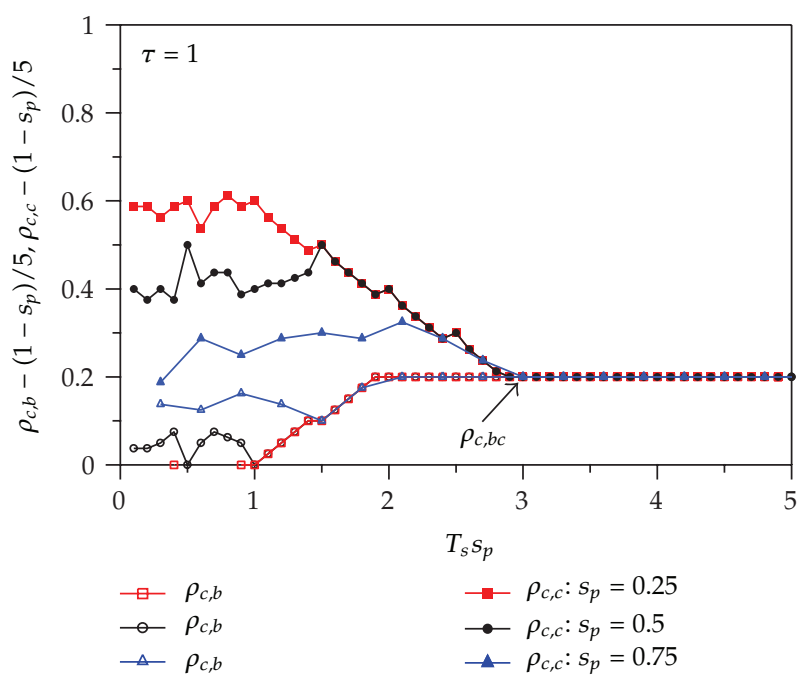

(b)

Figure 7: (a) Plots of the transition densities $\rho_{c, b}$ and $\rho_{c, c}$ against dimensionless cycle time at splits $s_{p}=0.25$, $0.5,0.75$ at $\tau=1.0$. (b) Plots of rescaled transition points $\rho_{c, b}-\left(1-s_{p}\right) / 5$ and $\rho_{c, c}-\left(1-s_{p}\right) / 5$ against rescaled dimensionless cycle time $T_{s} s_{p}$ for (a).

curve. Also, the transition points $\rho_{c, c}$ at $s_{p}=0.25,0.5,0.75$ collapse on a single curve. The transition points $\rho_{c, b}$ and $\rho_{c, c}$ scale as

$$
\begin{aligned}
& \rho_{c, b}=-\frac{1-s_{p}}{5}+g_{5}\left(T_{s} s_{p}\right), \\
& \rho_{c, c}=-\frac{1-s_{p}}{5}+g_{6}\left(T_{s} s_{p}\right),
\end{aligned}
$$

where $g_{5}(x)$ and $g_{6}(x)$ are the scaling functions for the two dynamic transitions. 


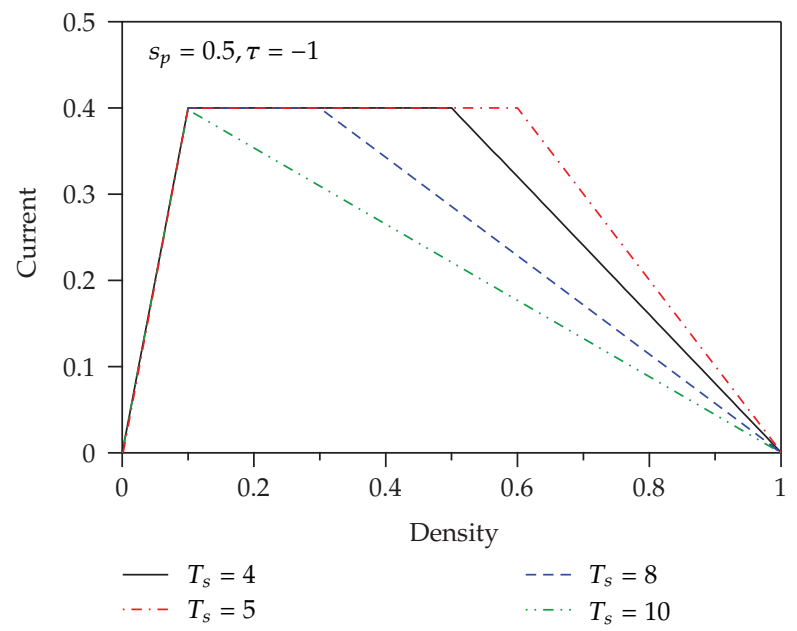

Figure 8: Fundamental diagrams at $\tau=-1.0$ for the dimensionless cycle times $T_{s}=4.0,5.0,8.0,10.0$.

We study the dependence of the transition points $\rho_{c, b}$ and $\rho_{c, c}$ on the dimensionless cycle time for various values of the offset time $\tau$. Figure 10(a) shows the plots of transition densities $\rho_{c, b}$ and $\rho_{c, c}$ against the dimensionless cycle time for $\tau=0.0,1.0,2.0,3.0$ at split $s_{p}=0.5$. Figure $10(\mathrm{~b})$ shows the plot of the rescaled transition density $\rho_{c, b}-\tau\left(1-s_{p}\right) / 5$ against the rescaled cycle time $T_{s} s_{p}-\tau$ for Figure 10(a). The transition points $\rho_{c, b}$ for $\tau=0.0,1.0,2.0$, 3.0 collapse on a single curve. Figure 10(c) shows the plot of the rescaled transition density $\rho_{c, c}-\tau\left(1-s_{p}\right) / 5$ against the rescaled cycle time $T_{s} s_{p}+\tau$ for Figure 10(a). The transition points $\rho_{c, c}$ for $\tau=0.0,1.0,2.0,3.0$ approach to a single curve. The transition points $\rho_{c, b}$ and $\rho_{c, c}$ scale for $0 \leq \tau \leq 3.0$ as

$$
\begin{aligned}
& \rho_{c, b}=\frac{\tau\left(1-s_{p}\right)}{5}+g_{b}\left(T_{s} s_{p}-\tau\right), \\
& \rho_{c, c}=\frac{\tau\left(1-s_{p}\right)}{5}+g_{c}\left(T_{s} s_{p}+\tau\right),
\end{aligned}
$$

where $g_{b}(x)$ and $g_{c}(x)$ are the scaling functions for the two dynamic transitions.

The transition points $\rho_{c, b}$ and $\rho_{c, c}$ scale for $-1.0 \leq \tau \leq 0.0$ as (3.5) and (3.6). The scaling forms (3.5) and (3.6) of transition points $\rho_{c, b}$ and $\rho_{c, c}$ hold only for $-1.0 \leq \tau \leq 3.0$. The scaling forms are not satisfied for the values of the dimensionless offset time except for $-1.0 \leq \tau \leq 3.0$ but the profiles of the transition points versus the dimensionless cycle time deviate from the scaling forms for $\tau<-1.0$ and $\tau>3.0$.

Thus, we obtain the following finding. The transition point from the undersaturated traffic to the saturated traffic scales as (3.5) for any values of split and offset time. Also, the transition point from the saturated traffic to the oversaturated traffic scales as (3.6) for any values of split and offset time. 


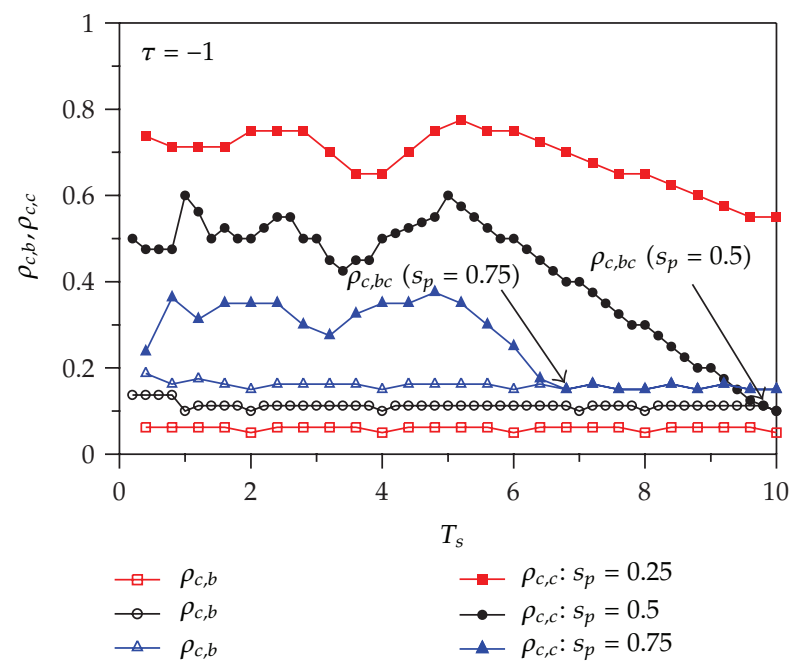

(a)

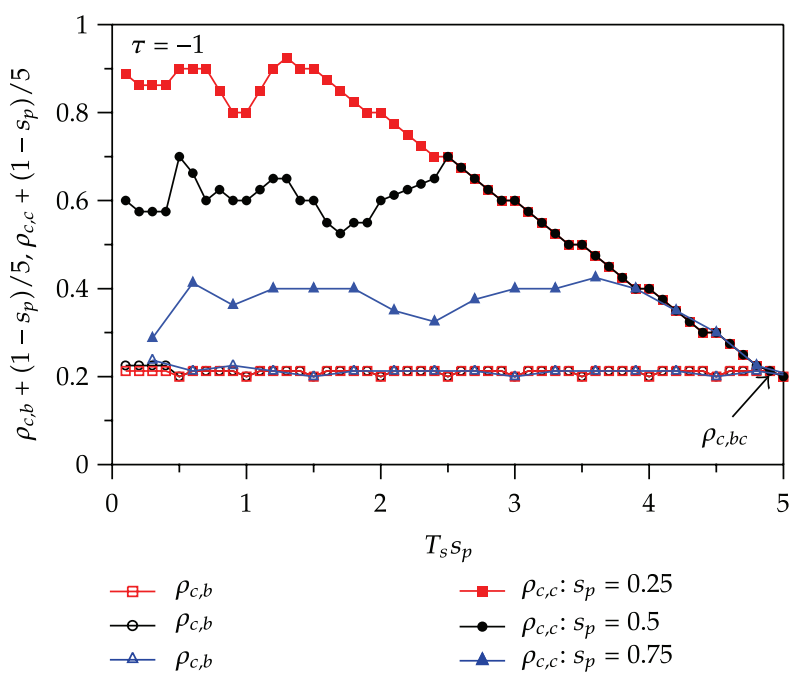

(b)

Figure 9: (a) Plots of the transition densities $\rho_{c, b}$ and $\rho_{c, c}$ against dimensionless cycle time at splits $s_{p}=$ $0.25,0.5,0.75$ at $\tau=-1.0$. (b) Plots of rescaled transition points $\rho_{c, b}+\left(1-s_{p}\right) / 5$ and $\rho_{c, c}+\left(1-s_{p}\right) / 5$ against rescaled dimensionless cycle time $T_{s} s_{p}$ for (a).

\section{Summary}

The deterministic cellular automaton model was presented for vehicular traffic through the series of traffic signals. The CA model is described by the difference equation. We have studied the effect of both vehicular density and signal's characteristics on dynamic behavior of vehicles by using the CA model. The fundamental diagram was derived for various values of the cycle time. The effect of the dimensionless cycle time on the fundamental diagram was clarified. It was found that the fundamental diagram changes from the typical trapezoid to the triangle with increasing the dimensionless cycle time. It was shown that the fundamental 


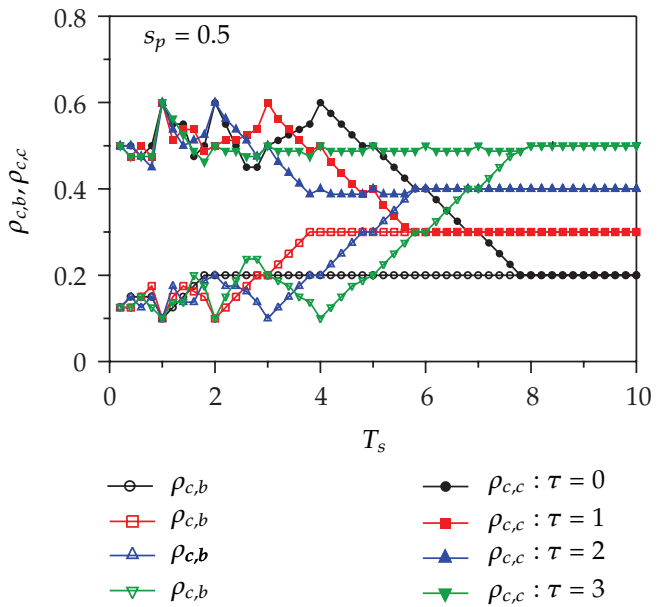

(a)

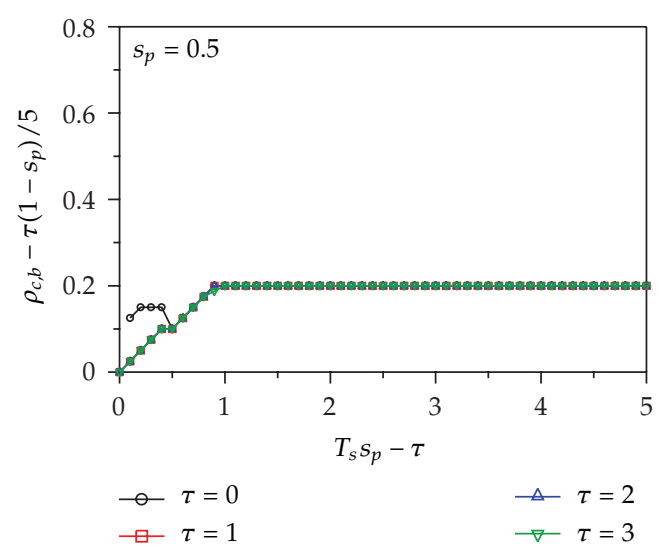

(b)

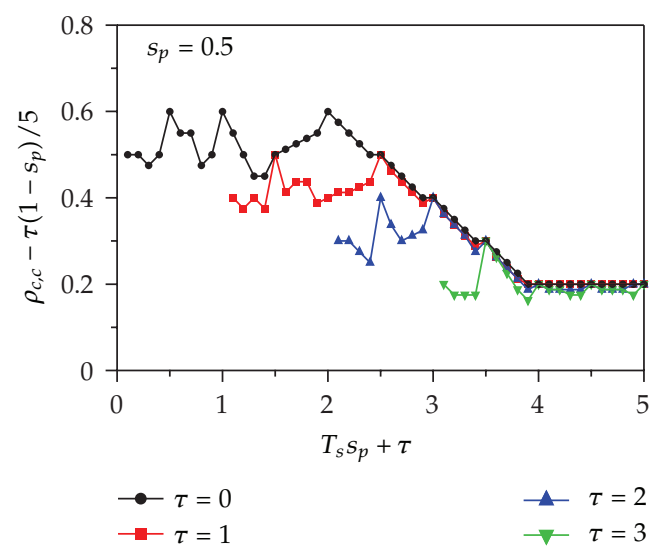

(c)

Figure 10: (a) Plots of transition densities $\rho_{c, b}$ and $\rho_{c, c}$ against the dimensionless cycle time for $\tau=0.0,1.0$, 2.0, 3.0 at split $s_{p}=0.5$. (b) Plot of the rescaled transition density $\rho_{c, b}-\tau\left(1-s_{p}\right) / 5$ against the rescaled cycle time $T_{s} s_{p}-\tau$ for (a). (c) Plot of the rescaled transition density $\rho_{c, c}-\tau\left(1-s_{p}\right) / 5$ against the rescaled cycle time $T_{s} s_{p}+\tau$ for (a).

diagram also depends highly on the split and the dimensionless offset time. It was found that the transition densities from the undersaturated traffic to the saturated traffic and from the saturated traffic to the oversaturated traffic scale as (3.5) and (3.6) for various values of both split and offset time.

The deterministic CA model proposed by this paper will be useful for studying the traffic flow through a series of signals controlled by the cycle time, the split, and the offset time because it is described by the difference equation. The study for the dependence of the dynamic transitions on the cycle time, the split, and the offset time is the first. Especially, the change of the fundamental diagram from the trapezoid to the triangle will be interesting. 


\section{References}

[1] T. Nagatani, "The physics of traffic jams," Reports on Progress in Physics, vol. 65, no. 9, article 1331, 2002.

[2] D. Helbing, "Traffic and related self-driven many-particle systems," Reviews of Modern Physics, vol. 73, no. 4, pp. 1067-1141, 2001.

[3] D. Chowdhury, L. Santen, and A. Schadscheider, "Statistical physics of vehicular traffic and some related systems," Physics Reports, vol. 329, no. 4-6, pp. 199-329, 2000.

[4] B. S. Kerner, The Physics of Traffic, Springer, Heidelberg, Germany, 2004.

[5] A. Kirchner and A. Schadschneider, "Simulation of evacuation processes using a bionics-inspired cellular automaton model for pedestrian dynamics," Physica A, vol. 312, no. 1-2, pp. 260-276, 2002.

[6] W. Wang, W. Zhang, H. Guo, H. Bubb, and K. Ikeuchi, "A safety-based approaching behavioural model with various driving characteristics," Transportation Research C, vol. 19, no. 6, pp. 1202-1214, 2011.

[7] G. H. Peng, X. H. Cai, C. Q. Liu, and B. F. Cao, "A new lattice model of traffic flow with the consideration of the driver's forecast effects," Physics Letters A, vol. 375, no. 22, pp. 2153-2157, 2011.

[8] T. Nagatani, "Chaotic jam and phase transition in traffic flow with passing," Physical Review E, vol. 60, no. 2, pp. 1535-1541, 1999.

[9] L. A. Safonov, E. Tomer, V. V. Strygin, Y. Ashkenazy, and S. Havlin, "Multifractal chaotic attractors in a system of delay-differential equations modeling road traffic," Chaos, vol. 12, no. 4, pp. 1006-1014, 2002.

[10] E. Brockfeld, R. Barlovic, A. Schadschneider, and M. Schreckenberg, "Optimizing traffic lights in a cellular automaton model for city traffic," Physical Review E, vol. 64, no. 5, Article ID 056132, 12 pages, 2001.

[11] D. W. Huang and W. N. Huang, "Traffic signal synchronization," Physical Review E, vol. 67, no. 5, Article ID 056124, 7 pages, 2003.

[12] M. Sasaki and T. Nagatani, "Transition and saturation of traffic flow controlled by traffic lights," Physica A, vol. 325, no. 3-4, pp. 531-546, 2003.

[13] S. Lammer and D. Helbing, "Self-control of traffic lights and vehicle flows in urban road networks," Journal of Statistical Mechanics: Theory and Experiment, vol. 2008, Article ID P04019, 2008.

[14] B. A. Toledo, V. Munoz, J. Rogan, and C. Tenreiro, "Modeling traffic through a sequence of traffic lights," Physical Review E, vol. 70, no. 1, Article ID 016107, 6 pages, 2004.

[15] B. A. Toledo, E. Cerda, J. Rogan et al., "Universal and nonuniversal features in a model of city traffic," Physical Review E, vol. 75, no. 2, Article ID 026108, 10 pages, 2007.

[16] T. Nagatani, "Self-similar behavior of a single vehicle through periodic traffic lights," Physica A, vol. 347, pp. 673-682, 2005.

[17] T. Nagatani, "Control of vehicular traffic through a sequence of traffic lights positioned with disordered interval," Physica A, vol. 368, no. 2, pp. 560-566, 2006.

[18] T. Nagatani, "Clustering and maximal flow in vehicular traffic through a sequence of traffic lights," Physica A, vol. 377, no. 2, pp. 651-660, 2007.

[19] T. Nagatani, "Effect of irregularity on vehicular traffic through a sequence of traffic lights," Physica A, vol. 387, no. 7, pp. 1637-1647, 2008.

[20] T. Nagatani, "Traffic states and fundamental diagram in cellular automaton model of vehicular traffic controlled by signals," Physica A, vol. 388, no. 8, pp. 1673-1681, 2009.

[21] C. Chen, J. Chen, and X. Guo, "Influences of overtaking on two-lane traffic with signals," Physica A, vol. 389, no. 1, pp. 141-148, 2010.

[22] M. Fukui and Y. Ishibashi, "Traffic flow in 1D cellular automaton model including cars moving with high speed," Journal of the Physical Society of Japan, vol. 65, pp. 1868-1870, 1996. 


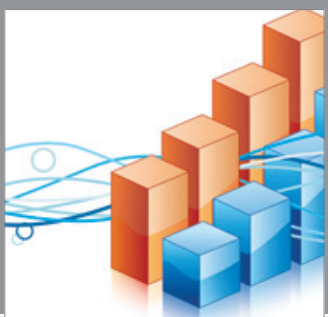

Advances in

Operations Research

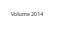

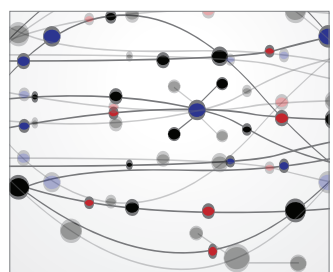

\section{The Scientific} World Journal
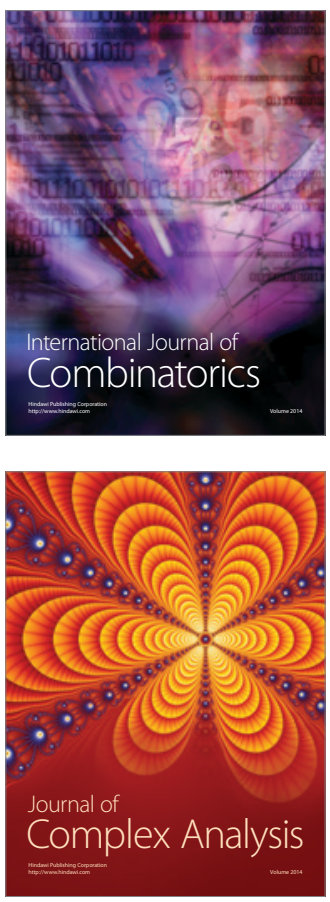

International Journal of

Mathematics and

Mathematical

Sciences
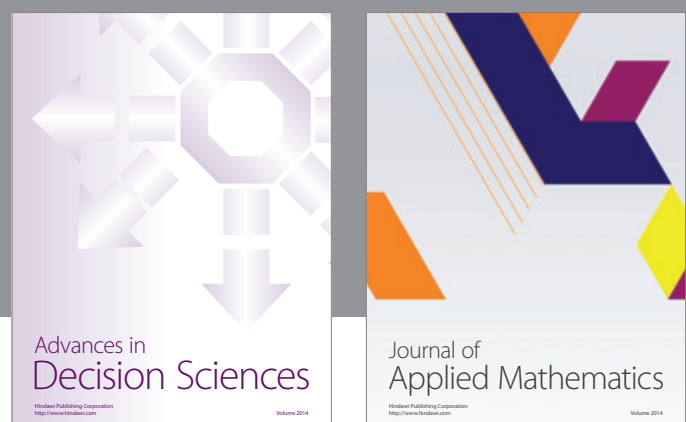

Journal of

Applied Mathematics
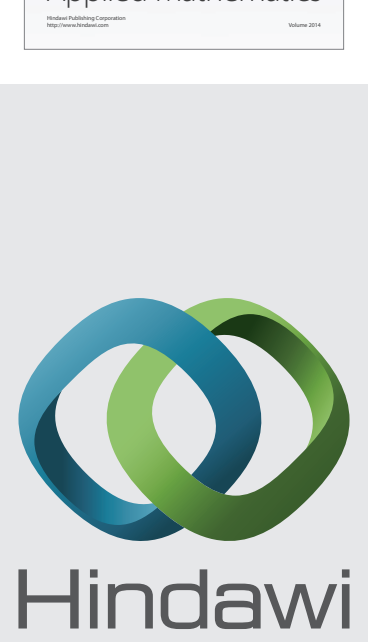

Submit your manuscripts at http://www.hindawi.com
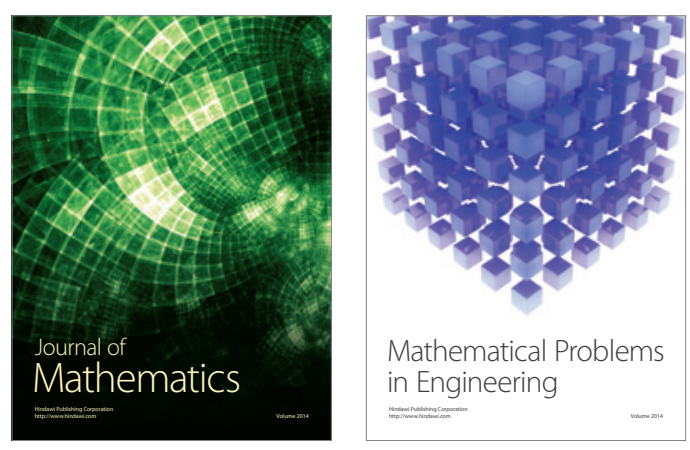

Mathematical Problems in Engineering
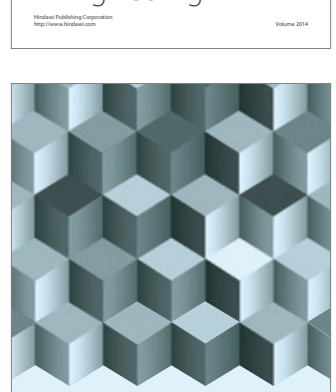

Journal of

Function Spaces
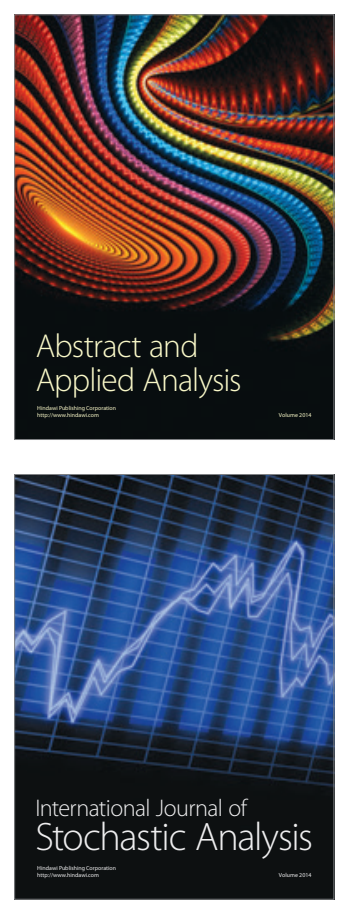

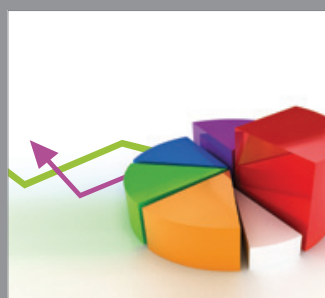

ournal of

Probability and Statistics

Promensencen
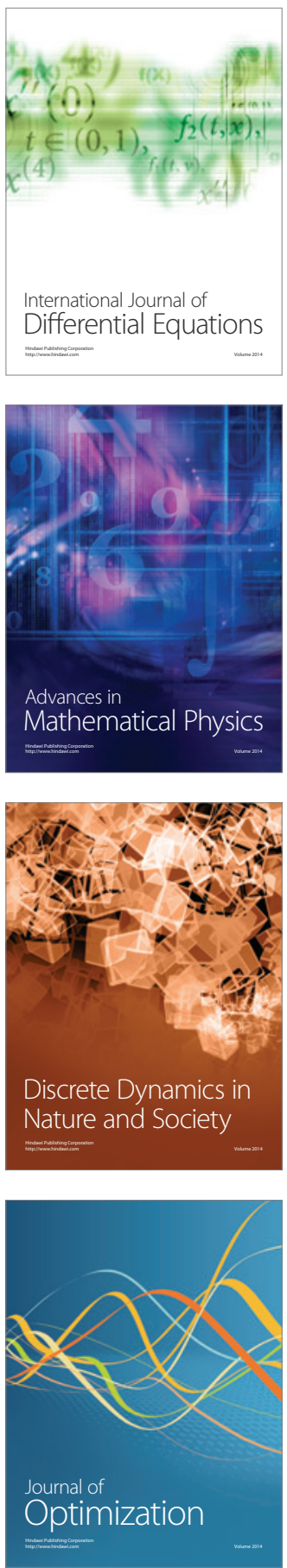